\title{
The falling rates of hospital admission, case fatality, and population-based mortality for subarachnoid hemorrhage in England, 1999-2010
}

\author{
Toqir K. Mukhtar, MSc, ${ }^{1}$ Andrew J. Molyneux, FRCR, ${ }^{2}$ Nick Hall, BSc, ${ }^{1}$ David R. G. Yeates, PhD, \\ Raphael Goldacre, BA, ${ }^{1}$ Mary Sneade, BA, ${ }^{2}$ Alison Clarke, BA, ${ }^{2}$ and Michael J. Goldacre, FFPH ${ }^{1}$

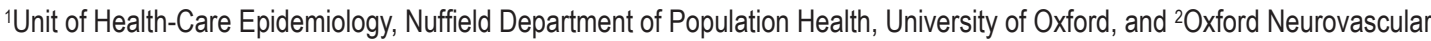
and Neuroradiology Research Unit, Nuffield Department of Surgical Sciences, John Radcliffe Hospital, University of Oxford, United Kingdom
\end{abstract}
OBJECTIVE In this study, the authors examined trends in population-based hospital admission rates, patient-level case fatality rates (CFRs), and population-based mortality rates for nontraumatic (spontaneous) subarachnoid hemorrhage $(\mathrm{SAH})$ in England.

METHODS Population-based admission and mortality data (59,599 people admitted to a hospital with SAH, 19992010; 37,836 people whose death certificates mentioned SAH, 1995-2010) were analyzed.

RESULTS Hospital admission rates for SAH per million population declined by $18.3 \%$, from 100.4 (95\% Cl 97.6-103.1) in 1999 to 82.0 (95\% Cl 79.7-84.4) in 2010. CFRs at less than 30 days per 100 patients decreased by $18.2 \%$, from 29.7 (95\% Cl 28.5-31.0) in 1999 to $24.3(95 \%$ Cl 23.2-25.5) in 2010. Population-based mortality rates per million population, where SAH was recorded as underlying cause of death on the death certificate, declined by $39.8 \%$, from $41.2(95 \% \mathrm{Cl}$ $39.5-43.0)$ in 1999 to $24.8(95 \%$ Cl 23.6-26.1) in 2010.

CONCLUSIONS Population-based hospital admission rates, patient-level CFRs, and population-based mortality rates all declined between 1999 and 2010. Part of the decline in mortality rates for SAH is likely to be attributable to a decline in incidence. It is also, in part, attributable to increased survival after SAH. The available data do not allow us to compare the effects of different treatment methods for SAH on case fatality and mortality. During the period of study, mortality rates declined by almost $40 \%$, and it is likely that there are a number of factors contributing to this substantial improvement in outcomes for SAH patients in England.

http://thejns.org/doi/abs/10.3171/2015.5.JNS142115

KEY WORDS subarachnoid hemorrhage; epidemiology; vascular disorders

$\mathrm{N}$ ONTRAUMATIC (spontaneous) subarachnoid hemorrhage (SAH) affects about 9 people per 100,000 person-years in most of Western Europe and the United States. ${ }^{1}$ Approximately $85 \%$ of these cases are due to rupture of an intracranial aneurysm. In patients who survive the initial rupture, and are suitable for active intervention, modern management is to secure the aneurysm responsible for the hemorrhage as promptly as reasonably possible, as the consequences of early aneurysm re-rupture are often catastrophic. The costs of treatment and care for dependent survivors represent a substantial burden to families, health care systems, and wider society. Accord- ingly, reductions in case fatality rates (CFRs) and dependency following treatment can have an important impact on all three.

Population-based mortality rates for $\mathrm{SAH}$ have declined in many developed countries, including the UK, over the past 4 decades..$^{3,4,8}$ Evidence from systematic reviews suggests that, during the same time period, population-based incidence rates for $\mathrm{SAH}$ have either remained stable, ${ }^{7}$ or there have been modest declines, with wide variation in rates between countries. ${ }^{1}$ There is conflicting evidence on trends in hospital admission rates for $\mathrm{SAH}$, with evidence from the US suggesting rates have remained stable, ${ }^{17}$ and

ABBREVIATIONS CFR = case fatality rate; GDC = Guglielmi detachable coil; HES = Hospital Episode Statistics; HSCIC = Health and Social Care Information Centre; ISAT = International Subarachnoid Aneurysm Trial; NCEPOD = National Confidential Enquiry into Patient Outcome and Death; NHS = National Health Service; RCS = Royal College of Surgeons; $\mathrm{SAH}=$ subarachnoid hemorrhage.

SUBMITTED September 10, 2014. ACCEPTED May 14, 2015.

INCLUDE WHEN CITING Published online January 1, 2016; DOI: 10.3171/2015.5.JNS142115. 
data from the UK pointing to a decline in hospital admission rates. ${ }^{19}$ There is also some evidence to show declines in CFRs for SAH both in the UK and the US..$^{13,16}$

Over the last 2 decades, there have been significant changes in the management of SAH in the UK, the US, and worldwide. In England, prior to 1992, neurosurgical clip ligation was the only option available to secure a ruptured aneurysm. In the US, the Guglielmi detachable coil (GDC) was first used to treat cerebral aneurysms in 1990, as part of an FDA investigational device exemption, and in the UK in 1992. Use of the GDC in Europe increased quite rapidly to treat high surgical risk aneurysms, such as those in the basilar location. In 1995, the GDC device was approved by the FDA for surgically high-risk intracranial aneurysms. Other improvements in the management of patients with SAH have occurred over the last 2 decades: improvements in neurointensive care, and in England, faster admission of patients to neurosurgical centers for aneurysm treatment. The introduction of computed tomography angiography (CTA) has expedited both the identification and treatment of patients with ruptured aneurysms. Operative neurosurgical care has also improved with the introduction of indocyanine green angiography and intraoperative ultrasound.

In 1994, the International Subarachnoid Aneurysm Trial (ISAT), a prospective randomized trial of endovascular coiling in the UK, commenced. It was funded by the UK Medical Research Council from 1997. Recruitment to the trial was halted by the Trial Steering Committee in 2002 following the release of unblinded data by the Data Monitoring Committee, which showed significant clinical benefit at 1 year in patients allocated to endovascular coiling compared with those allocated to craniotomy and neurosurgical clipping. The results of the trial were published in 2002 and 2005, and the findings of the trial informed and influenced routine clinical practice in the UK. ${ }^{9,10}$

In the present study, our objectives were to report on trends in population-based hospital admissions, patientlevel case fatality, and population-based mortality rates for SAH. We then investigated whether the declines in case fatality and population-based mortality might, in part, be attributable to changes of practice in respect of neurosurgical clipping and coil embolization.

\section{Methods \\ Data Sources}

Data on all hospital admissions in all National Health Service (NHS) hospitals in England (1999-2011) were obtained from the Hospital Episode Statistics (HES) database, supplied to us in files from the Health and Social Care Information Centre (HSCIC). The HSCIC has been unable to supply more recent data. All patients in England with SAH are admitted to an NHS district hospital and treated in an NHS neurosurgical unit. In mid-2011, the census-based population estimate for England was 53.11 million..$^{15}$ Data on successive admissions for each person were linked into a time-sequenced file for each individual, and these were also linked to the person's mortality record (if death had occurred). Mortality data for England (19952011) were obtained from death registration data supplied by the English Office for National Statistics. The earliest year in which the HES database can be reliably linked is 1999, and the earliest data available to us in which all certified causes of death are on each death certificate are for the year 1995. As some deaths that occur in each year are not registered until the following year, we examined data including those for 2011 registrations, but we confined our analyses of mortality to data for deaths that occurred in 2010 (to avoid end-year incompleteness of registrations). Our analyses of admission and CFRs were confined to admissions up to and including 2010 (to permit 365-day "follow up" into 2011 for those admitted in 2010).

Admission and mortality records were selected using codes 430 in the ninth revision and I60 in the tenth revision of the International Classification of Diseases. ${ }^{20}$ Procedures were selected from admissions records using code L33.2 for clipping of aneurysm of cerebral artery, and codes O01.1-O02.9 for coil embolization of an aneurysm, in the fourth revision of the Office of Population Census and Surveys Classification of Surgical Operations and Procedures..$^{14}$ Coding for coil embolization of an aneurysm, a newer procedure, was not available for the full period of study. Codes for coil embolization were first introduced in the HES database in 2006, although data for coiling are likely to be incomplete for that year. For this reason, we have limited our analyses on rates for coiling to 2007-2010. For any patient with more than one admission for $\mathrm{SAH}$, the first admission was selected for analysis.

\section{Statistical Analysis: Calculation of Rates}

Age-specific and age-standardized rates were calculated for rates of hospital admission, operative procedures, person-based case fatality, and population-based mortality. Admission rates, procedure rates, and mortality rates were standardized using the direct method, 5-year age groups, and the age distribution of the European standard population. CFRs in each year were age standardized using 5-year age groups and the age distribution of the population of patients admitted to a hospital for SAH in England in the years 1999-2010 combined as the standard. Trends in agestandardized hospital admissions, procedure, case fatality, and mortality rates were modeled using joinpoint regression (Joinpoint Desktop Software available at https://surveillance.cancer.gov/joinpoint/download). ${ }^{5,6,11}$

\section{Statistical Analysis: Joinpoint Analysis}

Joinpoint regression is a statistical technique for estimating the location of a significant change in the slope of a trend line. When modeling a series of annual rates, the location of an estimated significant change in trend, or joinpoint, will be a particular year. The log-linear option was used to model annual age-standardized hospital admission, procedure, case fatality, and mortality rates. The default number of joinpoints was allowed for each model (the default value changes as the number of data points increases), and the overall significance level for permutation tests was set at $\alpha=0.05$. A model of best fit was compared with more and/or less nonsignificant joinpoint models of the same series of rates. 


\section{Binary Logistic Regression}

Binary logistic regression analyses were used to calculate the odds of $<30$-day and $<365$-day case fatality.

\section{Results}

In the study period, there were 59,599 people admitted to hospital with SAH and 37,836 people whose death certificates mentioned SAH (as either the underlying or contributory cause on the death certificate).

\section{Trends in Hospital Admission Rates for SAH, 1999-2010}

Table 1 shows the decline in age-specific admission rates for SAH. Between 1999 and 2010, age-specific admission rates declined in all age groups, except for those aged 70 and over. For all ages combined, age-standardized rates fell from 100.4 per million in 1999 to 82.0 per million in 2010, an 18.3\% decline (Fig. 1A).

Joinpoint analyses showed that for all age groups combined (see Supplementary Table 1) admission rates declined significantly between 1999 and 2010, but without an estimated significant change in the scale of downward trend in specific years (i.e., there were no joinpoints on the trend line and, therefore, no significant acceleration or deceleration of the downward trend). Trend lines for the individual age groups $<40,40-49,50-59$, and 60-69 years all showed significant declines during the period of study, with no significant change in the downward trend in any particular year. For those 70 years of age and older, the annual percentage change $(0.3 \%)$ was not significant.

\section{Trends in Case Fatality Rates Following Hospital Admission for SAH, 1999-2010}

Table 2 shows the decline in age-standardized patientlevel CFRs for SAH. Between 1999 and 2010, < 30-day CFRs declined by $18.2 \%,<60$-day by $19.3 \%,<90$-day by $18.8 \%$, and < 365-day by $18.3 \%$. CFRs at less than 30 days were $29.7 \%$ in $1999,30.3 \%$ in 2005 , and $24.3 \%$ in 2010. CFRs under 365 days were $35.6 \%$ in $1999,36.9 \%$ in 2005 , and $29.1 \%$ in 2010 .

\section{Trends in Mortality Rates, 1995-2010}

We analyzed mortality based on underlying cause and all mentions on the death certificate. Supplementary Table 2 shows that declines in age-specific mortality rates were similar for underlying cause and for all mentions.

Between 1995 and 2010, the largest decline in mortality observed was for individuals under 40 years of age $(57.6 \%$ [all mentions]); during the same period, for all ages combined, mortality rates declined by $41.0 \%$ (all mentions).

Joinpoint analyses of age-standardized mortality rates (Supplementary Table 3) show that, for those under 40 years, an estimated change of trend occurred in 2006 for SAH as the underlying cause of death, with a significant acceleration of the downward trend in rates between Period 1 (1995-2006) and Period 2 (2006-2010). Measured as all mentions, there were significant accelerations of the downward trend for those aged 40-49 and 50-59 years in 2003, aged 60-69 in 1999, aged 70 and over in 2000, and for all ages in 2002. The changes in trend at these times were followed by significant acceleration in the decline in mortality.

For all ages combined, measured as underlying cause of death (Fig. 1B), the annual percentage decline for the period 2003-2010 was -6.3 (95\% CI -7.2 to -5.3$)$, which was more than 3 times greater than that for the period 1995-2003 (-2.0 [95\% CI -2.7 to -1.2$])$.

\section{Trends in Procedure Rates for Neurosurgical Clipping, 1999-2010}

Table 3 shows that age-standardized rates for neurosurgical clipping, all ages combined, dropped by $68.3 \%$, from 19.9 per million (95\% CI 18.6-21.1) to 6.3 per million (95\% CI 5.7-7.0) across the study period. Joinpoint analyses of these rates show estimated changes in trend in 2001 and 2005 (Fig. 1C). There were significant declines in all 3 periods of the trend line, with the steepest decline between 2001 and 2005, with an annual percentage change of $-18.9 \%$ (95\% CI -22.7 to -15.0$)$. This decline is more than twice the annual percentage decline in the period 1999-2001. The pattern of decline is broadly

TABLE 1. Age-specific admission rates for nontraumatic SAH per million population in England by year and age-group, males and females combined, 1999-2010

\begin{tabular}{ccccccc}
\hline & \multicolumn{5}{c}{ No. of SAH Per Million Population } \\
\cline { 2 - 6 } Yr & All Ages & $<40$ Yrs & $40-49$ Yrs & $50-59$ Yrs & $60-69$ Yrs & $\geq 70$ Yrs \\
\hline 1999 & 107.7 & 26.7 & 145.3 & 220.2 & 247.5 & 214.2 \\
\hline 2000 & 101.3 & 27.8 & 132.5 & 195.3 & 219.7 & 212.3 \\
\hline 2001 & 102.7 & 27.9 & 142.7 & 195.4 & 206.6 & 219.0 \\
\hline 2002 & 100.3 & 26.9 & 137.0 & 190.6 & 202.7 & 214.4 \\
\hline 2003 & 100.8 & 25.2 & 139.5 & 197.1 & 213.0 & 204.1 \\
\hline 2004 & 99.9 & 26.4 & 140.2 & 179.7 & 194.8 & 220.4 \\
\hline 2005 & 94.5 & 25.0 & 129.8 & 180.4 & 182.1 & 198.8 \\
\hline 2006 & 93.7 & 25.4 & 122.4 & 164.1 & 180.4 & 214.8 \\
\hline 2007 & 95.5 & 23.7 & 120.8 & 172.1 & 184.1 & 225.4 \\
\hline 2008 & 95.4 & 24.3 & 122.4 & 167.7 & 176.2 & 227.5 \\
\hline 2009 & 95.8 & 21.8 & 119.5 & 174.6 & 177.2 & 233.6 \\
\hline 2010 & 94.9 & 21.3 & 122.0 & 174.1 & 175.6 & 223.3 \\
\hline
\end{tabular}



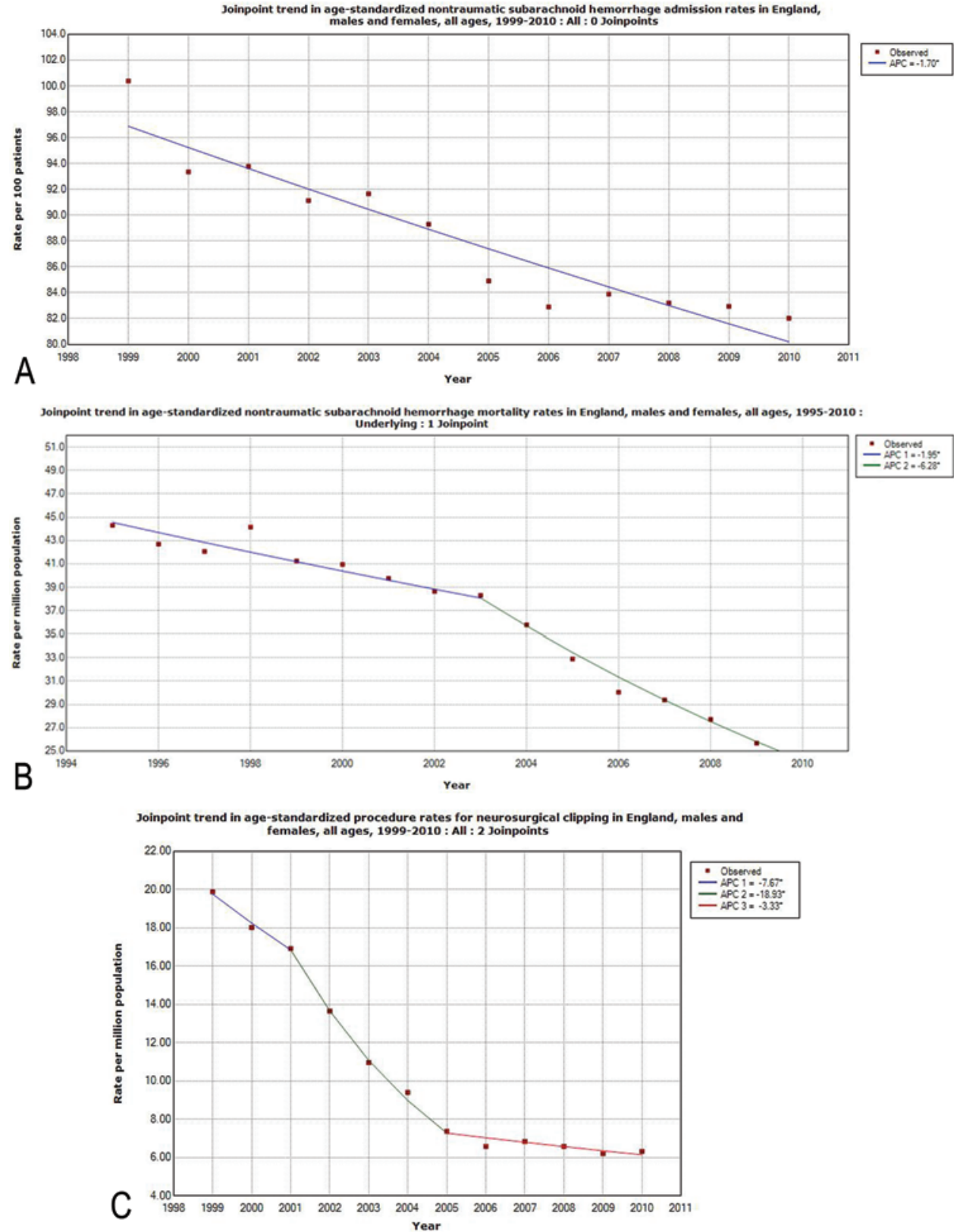

FIG. 1. Joinpoint trends for SAH admission, mortality, and neurosurgical clipping rates for males and females of all ages in England. A: Trend in age-standardized nontraumatic SAH admission rates from 1999 to 2010. All: 0 joinpoints. B: Trend in nontraumatic SAH mortality rates from 1995 to 2010 . Underlying: 1 joinpoint. C: Trend in age-standardized procedure rates for neurosurgical clipping from 1999 to 2010. All: 2 joinpoints. APC = annual percentage change. Figure is available in color online only.

similar to that for all-ages mortality, measured as all mentions, where there was a significant downward change in trend in 2002, and where the percentage decline in Period 2 (2002-2010) was more than 3 times the rate of decline in Period 1 (1995-2002), see Supplementary Table 3.

\section{Trends in Procedure Rates for Coil Embolization, 2007-2010}

Data on coiling were not available for the full period of the study. Table 4 shows that age-standardized rates for coiling increased 14.3\% between 2007 and 2010. The total number of coded procedures (clipping \& coiling) in- creased from 1392 in 2007 to 1537 in 2010, an increase of $10.4 \%$.

\section{Scatterplots}

Supplementary Fig. 1A shows a scatterplot of age-standardized < 30-day CFRs and age-standardized procedure rates for neurosurgical clipping (1999-2010), and Supplementary Fig. 1B shows a scatterplot of age-standardized $<30$-day CFRs and age-standardized procedure rates for coil embolization (2007-2010). The data do not meet the required assumptions of the Pearson's product-moment correlation coefficient nor the Spearman's rank correla- 
TABLE 2. Age-standardized case fatality rates for nontraumatic SAH per 100 patients in England by time from hospital admission, males and females combined, 1999-2010*

\begin{tabular}{cccccc}
\hline & & \multicolumn{4}{c}{ Age-Standardized Case Fatality Rates (95\% Cl) } \\
\cline { 3 - 6 } Yr & No. of Admissions & $<30$ Days & $<60$ Days & $<90$ Days & $<365$ Days \\
\hline 1999 & 5283 & $29.7(28.5-31.0)$ & $32.1(30.9-33.3)$ & $33.0(31.8-34.2)$ & $35.6(34.4-36.9)$ \\
\hline 2000 & 4985 & $31.4(30.1-32.7)$ & $34.0(32.7-35.3)$ & $34.9(33.6-36.2)$ & $37.1(35.8-38.4)$ \\
\hline 2001 & 5077 & $31.2(29.9-32.4)$ & $33.7(32.4-34.9)$ & $34.7(33.4-35.9)$ & $37.0(35.8-38.3)$ \\
\hline 2002 & 4982 & $31.3(30.1-32.6)$ & $33.4(32.2-34.7)$ & $34.4(33.1-35.6)$ & $37.0(35.7-38.2)$ \\
\hline 2003 & 5027 & $31.5(30.3-32.8)$ & $33.7(32.4-34.9)$ & $34.2(32.9-35.4)$ & $36.1(34.8-37.4)$ \\
\hline 2004 & 5007 & $31.5(30.3-32.8)$ & $33.8(32.5-35.0)$ & $34.6(33.3-35.8)$ & $36.9(35.6-38.2)$ \\
\hline 2005 & 4768 & $30.3(29.0-31.5)$ & $32.3(31.0-33.5)$ & $33.1(31.9-34.4)$ & $36.9(35.6-38.2)$ \\
\hline 2006 & 4755 & $28.2(27.0-29.5)$ & $29.8(28.6-31.1)$ & $30.7(29.5-31.9)$ & $33.2(32.0-34.5)$ \\
\hline 2007 & 4882 & $27.7(26.5-29.0)$ & $29.3(28.1-30.5)$ & $30.3(29.0-31.5)$ & $33.0(31.7-34.2)$ \\
\hline 2008 & 4910 & $25.9(24.8-27.1)$ & $28.0(26.8-29.2)$ & $28.8(27.6-30.0)$ & $31.2(29.9-32.4)$ \\
\hline 2009 & 4965 & $25.2(24.0-26.3)$ & $26.8(25.7-28.0)$ & $27.7(26.5-28.9)$ & $30.6(29.4-31.8)$ \\
\hline 2010 & 4958 & $24.3(23.2-25.5)$ & $25.9(24.8-27.1)$ & $26.8(25.6-28.0)$ & $29.1(27.9-30.3)$ \\
\hline
\end{tabular}

* The deaths within 60,90 , and 365 days include all those in each preceding time interval.

tion coefficient. We further examine the relationship between CFRs and procedure rates for coiling or clipping by conducting binary logistic regression analyses.

\section{$<30$-Day and $<365-$ Day Case Fatality, 2007-2010}

Univariate analyses in Table 5 show that clipping (referent: coiling) and age were both significant predictors of $<30$-day and $<365$-day case fatality. Adjusted odds ratios in Table 5 show that the odds of case fatality $<30$ days and $<365$ days were significantly higher for patients treated with neurosurgical clipping compared with those treated with coil embolization. We were unable to adjust for casemix in these analyses.

\section{Relative Risk of Death, 2007-2010}

The relative risk of death (RR) within 30 days of hospital admission was significantly higher for patients who underwent clipping, 1.31 (95\% CI 1.09-1.59), compared

TABLE 3. Age-standardized procedure rates for neurosurgical clip ligation per million population in England, males and females combined, 1999-2010

\begin{tabular}{ccc}
\hline $\mathrm{Yr}$ & No. of Procedures & Rate of Clip Ligation $(95 \% \mathrm{Cl})$ \\
\hline 1999 & 966 & $19.9(18.6-21.1)$ \\
\hline 2000 & 887 & $18.0(16.8-19.2)$ \\
\hline 2001 & 843 & $16.9(15.8-18.1)$ \\
\hline 2002 & 684 & $13.7(12.6-14.7)$ \\
\hline 2003 & 557 & $11.0(10.0-11.9)$ \\
\hline 2004 & 484 & $9.4(8.6-10.2)$ \\
\hline 2005 & 379 & $7.4(6.6-8.1)$ \\
\hline 2006 & 345 & $6.6(5.9-7.3)$ \\
\hline 2007 & 360 & $6.8(6.1-7.6)$ \\
\hline 2008 & 349 & $6.6(5.9-7.3)$ \\
\hline 2009 & 335 & $6.2(5.5-6.9)$ \\
\hline 2010 & 340 & $6.3(5.7-7.0)$ \\
\hline
\end{tabular}

with patients who underwent coil embolization. The RR within 365 days of hospital admission for those treated with clipping compared with those who underwent coiling was also significantly higher, at 1.34 (95\% CI 1.14-1.57). We were unable to adjust for case-mix in these analyses.

\section{Discussion}

\section{The Decline in Hospital Admission Rates}

Between 1999 and 2010, age-standardized hospital admission rates for SAH per million population declined $18.3 \%$ in England. The reasons for this decline are unclear. A reduction in smoking rates, particularly in middle-aged females (the group most commonly affected by SAH), together with improved management of hypertension, are likely the most important factors contributing to the observed decline. It is unlikely to be due to an increase in the rate of patients dying from SAH before being admitted to hospital. This was not observed in two prospective population-based studies, the Oxford Community Stroke Project (1981-1986) and the Oxford Vascular Study (2002-2008), both based in Oxfordshire, England; in these two studies, only 3 of 65 patients died before reaching the hospital. ${ }^{8}$ A significant decline in incidence rates for SAH was not observed in the interval between these two studies; however, the number of events was small (65), because these studies were based on small populations. On the other hand, a national study of admissions for SAH in England

TABLE 4. Age-standardized procedure rates for coil embolization per million population in England, males and females combined, 2007-2010

\begin{tabular}{ccc}
\hline $\mathrm{Yr}$ & No. of Procedures & Rate of Coil Embolization $(95 \% \mathrm{Cl})$ \\
\hline 2007 & 1032 & $18.9(17.7-20.0)$ \\
\hline 2008 & 1145 & $20.8(19.6-22.0)$ \\
\hline 2009 & 1168 & $21.0(19.8-22.2)$ \\
\hline 2010 & 1197 & $21.6(20.3-22.8)$ \\
\hline
\end{tabular}


TABLE 5. Univariate and multivariate analyses of $<30$-day and $<365$-day case fatality, 2007-2010*

\begin{tabular}{lll}
\hline \multirow{2}{*}{ Analysis \& Factor } & \multicolumn{2}{c}{ Odds Ratio (95\% Cl) } \\
\cline { 2 - 3 } Univariate analysis & \multicolumn{3}{c}{$<365$ Days } & \\
\hline Male sex (referent female) & $0.92(0.75-1.12)$ & $1.01(0.85-1.20)$ \\
\hline Age & $1.02 \dagger(1.01-1.02)$ & $1.03 \dagger(1.03-1.04)$ \\
\hline Clipping (referent coiling) & $1.32 \dagger(1.06-1.63)$ & $1.34 \dagger(1.11-1.61)$ \\
\hline Yr of admission & $0.99(0.91-1.08)$ & $1.01(0.94-1.09)$ \\
\hline Multivariate analysis & & \\
\hline Clipping (referent coiling) & $1.36(1.10-1.68) \dagger \ddagger$ & $1.44(1.19-1.73) \dagger \ddagger$ \\
\hline
\end{tabular}

* These analyses exclude data for 104 patients who had codes for both clipping and coiling.

† Odds differ significantly from 1 .

$\ddagger$ Adjusted for age and sex.

showed that the number of admissions for SAH has declined over the past 13 years, from 7054 in 1998-1999 to 6166 in 2010-2011. ${ }^{19}$

\section{Case Fatality Rates, Mortality Rates, and Changes in Clinical Practice}

Between 1999 and 2010, age-standardized < 30-day CFRs per 100 patients decreased $18.2 \%$, and age-standardized population-based mortality rates per million population where SAH was recorded as the underlying cause of death declined $39.8 \%$. Our results support findings from a meta-analysis ${ }^{13}$ and a systematic review ${ }^{8}$ that both show that case fatality rates for SAH have declined. Our findings on mortality support those of previous studies, which indicate that population-based mortality for SAH has declined since the 1980s. ${ }^{3,4,8}$

There may be several reasons for beneficial trends in case fatality and population-based mortality rates in England. The urgent transfer of all SAH patients to neurosurgical care (most patients in England are admitted to a district general hospital without a neurosurgical service, and are transferred to the neurosurgical center after a diagnosis of SAH) is now standard care. In the past, there had been delays in the admission of some patients. A reduction of delays in transfer and aneurysm treatment is likely to have reduced the number of patients with early rebleeding, which, in turn, is likely to have reduced case fatality. It is also probable that more elderly patients and poor-grade patients, who may not have been deemed suitable for clipping, underwent coiling to secure the aneurysm.

Between 1999 and 2010, procedure rates for neurosurgical clipping in England declined by $68.3 \%$. The pattern of decline was similar to that observed for mortality. Procedure rates for neurosurgical clipping showed an estimated acceleration in decline in 2001, and mortality rates measured as mentions accelerated downward in 2002, as did mortality rates as underlying cause in 2003. The decline in rates per year after each of these changes in trend in Period 2 was more than twice the annual percentage change before the change in trend in Period 1. Reliable year-onyear data on national rates of coiling are not available from the HES database for the full run of years covered by our study. However, a study by the Royal College of Surgeons (RCS) in England showed that the decline in populationbased mortality rates coincided with an increase in the use of coil embolization after the recognition of the clinical benefit of coiling shown in the ISAT. ${ }^{18}$ A national study of SAH management in neurological and neuroscience centers in the UK by the National Confidential Enquiry into Patient Outcome and Death (NCEPOD) showed that in patients undergoing treatment for aneurysmal $\mathrm{SAH}, 84 \%$ had undergone coiling, $14 \%$ clipping, and $2 \%$ clipping after coiling had been attempted..$^{12}$ While codes for coil embolization of an aneurysm were not available for the full period of study, which is a shortcoming of the dataset, there is no evidence to suggest that patients who would have been suitable for treatment of an aneurysm after $\mathrm{SAH}$, and who did not undergo clipping, were left untreated; given the findings of the RCS and NCEPOD studies, it is reasonable to assume that most of these patients underwent coiling.

We used the only available study design that allowed for the investigation of whole-population trends in admission and mortality rates for SAH. It is not a design best suited for comparing the outcomes of clipping versus those of coiling; this is best done, and has been done, in randomized controlled trials. ${ }^{9,10}$

\section{Strengths and Limitations}

The strengths of the study are that we used a very large, national (and therefore representative) record-linked dataset, allowing us to take account of multiple admissions for each patient and to identify deaths whenever and wherever they occurred. The data also included all certified causes of death, allowing us to compare trends for mortality where SAH is recorded as the underlying cause of death with trends where SAH is mentioned as a contributory cause.

One potential limitation is that there were changes in rules for the coding of underlying cause of death in England in 2001, which could have affected mortality trends for $\mathrm{SAH}$, as has occurred for some other diseases. ${ }^{2,3}$ In fact, for SAH, the analysis of mentions showed that these rates were very similar to those shown for underlying cause. Another limitation is that routinely collected hospital data can be subject to errors such as miscoding of the disease classification, but the coding of SAH is very straightforward, and, providing the clinical diagnosis is given in the patient's case notes, coding errors should be uncommon. Another limitation that cannot be remedied in historical anonymized datasets are possible errors and omissions in recording and coding the operative procedures of clipping and coiling.

\section{Conclusions}

In England, between 1999 and 2010, hospital admission rates for SAH declined $18.3 \%,<30$-day CFRs for SAH declined $18.2 \%$, and population-based mortality rates for SAH declined 39.8\%. Part of the decline in populationbased mortality rates for SAH is likely to be attributable to a decline in incidence. It is also, in part, attributable to increased survival after SAH. The available data do not allow us to compare the effects of different treatment meth- 
ods for SAH on case fatality and mortality. During the period of study, mortality rates declined by almost $40 \%$, and it is likely that there are a number of factors contributing to this substantial improvement in outcomes for $\mathrm{SAH}$ patients in England.

\section{Acknowledgments}

The construction of the linked dataset was funded by the English National Institute for Health Research. This study was funded, in part, by the UK Medical Research Council. The funding sources had no influence over the study design, data analysis, data interpretation, report writing, or the decision to submit the paper for publication. T.K.M had full access to the data for this study, and all authors had final responsibility for the decision to submit the manuscript for publication. M.J.G and R.G. are funded, in part, by Public Health England.

\section{References}

1. de Rooij NK, Linn FHH, van der Plas JA, Algra A, Rinkel GJE: Incidence of subarachnoid haemorrhage: a systematic review with emphasis on region, age, gender and time trends. J Neurol Neurosurg Psychiatry 78:1365-1372, 2007

2. Goldacre MJ, Duncan ME, Cook-Mozaffari P, Griffith M: Trends in mortality rates comparing underlying-cause and multiple-cause coding in an English population 1979-1998. J Public Health Med 25:249-253, 2003

3. Goldacre MJ, Duncan M, Griffith M, Rothwell PM: Mortality rates for stroke in England from 1979 to 2004: trends, diagnostic precision, and artifacts. Stroke 39:2197-2203, 2008

4. Ingall TJ, Whisnant JP, Wiebers DO, O'Fallon WM: Has there been a decline in subarachnoid hemorrhage mortality? Stroke 20:718-724, 1989

5. Kim HJ, Fay MP, Feuer EJ, Midthune DN: Permutation tests for joinpoint regression with applications to cancer rates. Stat Med 19:335-351, 2000

6. Kim HJ, Feuer EJ, Barret MJ: Is the Trend Changing? Joinpoint Regression Analysis. Presented at the National Center for Health Statistics, Hyattsville, Maryland, 2012. (http://surveillance.cancer.gov/joinpoint/presentations.html) [Access September 16, 2015]

7. Linn FH, Rinkel GJ, Algra A, van Gijn J: Incidence of subarachnoid hemorrhage: role of region, year, and rate of computed tomography: a meta-analysis. Stroke 27:625-629, 1996

8. Lovelock CE, Rinkel GJ, Rothwell PM: Time trends in outcome of subarachnoid hemorrhage: Population-based study and systematic review. Neurology 74:1494-1501, 2010

9. Molyneux A, Kerr R, Stratton I, Sandercock P, Clarke M, Shrimpton J, et al: International Subarachnoid Aneurysm Trial (ISAT) of neurosurgical clipping versus endovascular coiling in 2143 patients with ruptured intracranial aneurysms: a randomised trial. Lancet 360:1267-1274, 2002

10. Molyneux AJ, Kerr RS, Yu LM, Clarke M, Sneade M, Yarnold JA, et al: International subarachnoid aneurysm trial (ISAT) of neurosurgical clipping versus endovascular coiling in 2143 patients with ruptured intracranial aneurysms: a randomised comparison of effects on survival, dependency, seizures, rebleeding, subgroups, and aneurysm occlusion. Lancet 366:809-817, 2005

11. Mukhtar TK, Yeates DG, Goldacre MJ: Breast cancer mortality trends in England and mammography screening (19712009): authors' response. J R Soc Med 107:54-55, 2014

12. National Confidential Enquiry into Patient Outcome and Death: Managing the Flow? A Review of the Care Received by Patients Who Were Diagnosed with an Aneurysmal Subarachnoid Haemorrhage. (http://www.
ncepod.org.uk/2013report2/downloads/Managing\%20the $\% 20$ Flow_FullReport.pdf) [Accessed September 9, 2015]

13. Nieuwkamp DJ, Setz LE, Algra A, Linn FH, de Rooij NK, Rinkel GJ: Changes in case fatality of aneurysmal subarachnoid haemorrhage over time, according to age, sex, and region: a meta-analysis. Lancet Neurol 8:635-642, 2009

14. Office of Population Censuses and Surveys: OPCS Classification of Interventions and Procedures, Version 4.6. London: National Health Service, 2011

15. Office for National Statistics: Population Estimates for England and Wales, Mid-2011 (2011 Census-Based). (http://www.ons.gov.uk/ons/rel/pop-estimate/populationestimates-for-england-and-wales/mid-2011--2011-censusbased-/index.html) [Accessed September 9, 2015]

16. Qureshi AI, Vazquez G, Tariq N, Suri MF, Lakshminarayan K, Lanzino G: Impact of International Subarachnoid Aneurysm Trial results on treatment of ruptured intracranial aneurysms in the United States. Clinical article. J Neurosurg 114:834-841, 2011

17. Rincon F, Rossenwasser RH, Dumont A: The epidemiology of admissions of nontraumatic subarachnoid hemorrhage in the United States. Neurosurgery 73:217-222, 212-213, 2013

18. Royal College of Surgeons of England: National Study of Subarachnoid Haemorrhage: Final Report. (https://www. rcseng.ac.uk/publications/docs/nat_study_subarachnoid_ haem_feb2006.html) [Accessed September 9, 2015]

19. Shiue I: Patterns of subarachnoid hemorrhage admissions in England, 2008-2011. Eur Neurol 69:242-245, 2013

20. World Health Organization: International Classification of Diseases. Manual of the International Statistical Classification of Diseases, Injuries, and Causes of Death, Tenth Revision. Geneva: World Health Organization, 1995, Vol. 1

\section{Disclosures}

A.J.M reports receiving personal fees from Sequent Medical Inc. as a consultant; grants from the UK Medical Research Council, outside the submitted work; and expert medical evidence in respect to cases of SAH. A.C. reports performing contract research for Sequent Medical Inc.

\section{Author Contributions}

Conception and design: Mukhtar, Molyneux, M Goldacre. Acquisition of data: Mukhtar, Hall, Yeates, R Goldacre. Analysis and interpretation of data: Mukhtar, Molyneux, Sneade, Clarke, M Goldacre. Drafting the article: Mukhtar. Critically revising the article: Mukhtar, Molyneux, M Goldacre. Reviewed submitted version of manuscript: all authors. Approved the final version of the manuscript on behalf of all authors: Mukhtar. Statistical analysis: Mukhtar, Yeates. Study supervision: Mukhtar, M Goldacre.

\section{Supplemental Information Online-Only Content}

Supplemental material is available with the online version of the article.

Supplementary Figures. http://thejns.org/doi/suppl/10.3171/ 2015.5.JNS142115.

Supplementary Tables. http://thejns.org/doi/suppl/10.3171/ 2015.5.JNS142115.

\section{Correspondence}

Toqir K. Mukhtar, Nuffield Department of Primary Care, University of Oxford, Radcliffe Observatory Quarter, Woodstock Rd., Oxford OX2 6GG, United Kingdom. email: toqir.mukhtar@phc. ox.ac.uk. 